\title{
Étude de la désorption laser UV d'une surface de silicium
}

\author{
T. Gonthiez, T. Gibert et P. Brault \\ Groupe de Recherche sur l'Énergétique des Milieux lonisés, Université d'Orléans, BP. 6744, \\ 45067 Orléans cedex 2, France
}

\begin{abstract}
Résumé : La désorption laser UV $\left(\lambda=355 \mathrm{~nm} ; \tau_{\mathrm{p}}=7,5 \mathrm{~ns}\right)$ d'une surface de silicium est étudiée dans un domaine de fluence allant de 300 à $700 \mathrm{~mJ} / \mathrm{cm}^{2}$. Nous réalisons l'étude des atomes neutres désorbés en couplant l'ionisation laser résonnante à la spectrométrie de masse. Le dispositif expérimental permet de mesurer des distributions de temps de vol dont l'interprétation nous renseigne sur la dynamique de désorption et sur les mécanismes de l'interaction laser UV/surface de silicium.
\end{abstract}

\section{INTRODUCTION}

Les couches minces de nitrure de silicium sont largement utilisées comme isolant et couche de passivation en micro-électronique. La nécessité de comprendre les mécanismes élémentaires de croissance de ces couches nitrurées conduit à étudier la réactivité spontanée et photoinduite de gaz azotés $\left(\mathrm{NH}_{3}, \mathrm{~N}_{2}\right)$ avec une surface de silicium. Pour optimiser le diagnostic par la désorption laser, il nous faut comprendre et contrôler les phénomènes intervenant lors de l'interaction laser avec une surface de silicium vierge. Des études déjà menées sur ce sujet [1-3] ont révélées des ambiguïtés sur les mécanismes physiques de la désorption. Ces études sont principalement basés sur l'analyse des ions.

Nous présentons les premiers résultats obtenus par notre équipe sur la désorption laser du silicium par l'analyse des atomes neutres par post-ionisation laser.

\section{DISPOSITIF EXPERIMENTAL}

Les expériences sont réalisées dans une enceinte à ultravide (pression: $8.10^{-10} \mathrm{mbar}$ ). Un laser Nd-YAG triplé en fréquence (Quantel, Brilliant- $\lambda=355 \mathrm{~nm}, \tau_{\mathrm{p}}=4,1 \mathrm{~ns}, \mathrm{f}=10 \mathrm{~Hz}$ ) irradie une surface de silicium (100) $(\rho=5-8 \Omega . \mathrm{cm}$, dopé en phosphore, $\mathrm{e}=0.5 \mathrm{~mm})$ avec des fluences allant de 300 à $700 \mathrm{~mJ} / \mathrm{cm}^{2}$. Les produits désorbés sont détectés par spectrométrie de masse soit directement dans le cas des ions ou après post-ionisation laser résonnante dans le cas des neutres. Un schéma d'ionisation par absorption de $(1+1)$ photons via l'étape résonnante entre les états $3 \mathrm{p}^{2}{ }^{3} \mathrm{P}\left(0 \mathrm{~cm}^{-1}\right)-4 \mathrm{~s} ! \mathrm{P}^{0}\left(40992 \mathrm{~cm}^{-1}\right)$ du silicium permet de sonder efficacement les atomes neutres. Le faisceau accordable en longueur d'onde $\lambda$ est issu d'un laser à colorant. Le domaine de longueur d'onde utile est obtenu par mélange entre la seconde harmonique d'un laser à colorant (mélange Rh610 et DCM, $\lambda=315,662 \mathrm{~nm}, \tau_{\mathrm{p}}=7,5 \mathrm{~ns}$ ) et le fondamental du laser de pompe (Nd-YAG, $\lambda=1064 \mathrm{~nm}, \tau_{\mathrm{p}}=7,5 \mathrm{~ns}, \mathrm{f}=10 \mathrm{~Hz}$ ).

Par ajustement du délai entre le faisceau de désorption et le faisceau sonde, nous pouvons effectuer des mesures de temps de vol (TOF) des atomes neutres de silicium au dessus de la surface. Pour plus de précisions dans l'interprétation chaque courbe de temps de vol est effectuée à trois hauteurs de faisceau sonde différentes $(2,35 ; 3,4$ et $4,25 \mathrm{~mm})$.

\section{RESULTATS}

\subsection{Photoionisation laser résonnante des espèces neutres de Si}

Les atomes $\mathrm{Si}^{\circ}$ sont sondés dans leur état fondamental ${ }^{3} \mathrm{P}$ par un schéma d'ionisation à $(1+1)$ photons impliquant la transition résonnante $3 \mathrm{p}^{2}{ }^{3} \mathrm{P}-4 \mathrm{~s}{ }^{1} \mathrm{P}^{0}$ à $\lambda_{\text {theor }}=243.877 \mathrm{~nm}$ (cf Figure 1-(a)). La figure 1-b montre le spectre d'ionisation de $\mathrm{Si}^{\circ}$ mesuré lorsque l'on fait varier continûment la longueur d'onde du laser accordable autour de la résonance. L'écart en longueur d'onde entre les valeurs théoriques et expérimentales n'est dû qu'à la calibration du laser à colorant. Les densités de puissance laser sonde varient de 1,5 à $4 \mathrm{MW} / \mathrm{cm}^{2}$ et permettent uniquement la saturation de l'étape d'absorption et non celle de l'étape d'ionisation. 


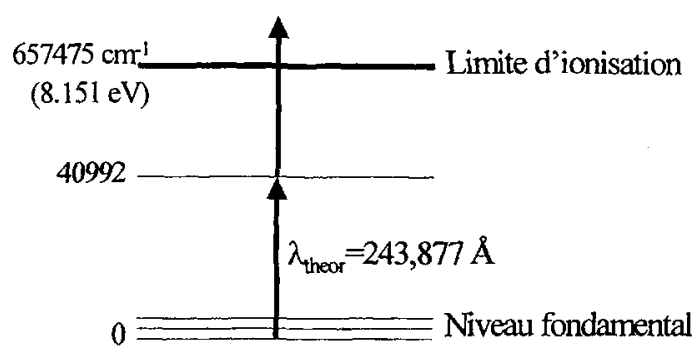

(a)

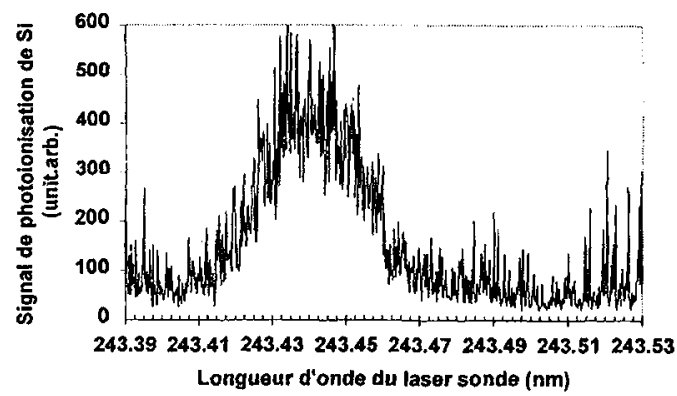

(b)

Figure 1. Photoionisation laser résonnante des atomes de $\mathrm{Si}^{\circ}:(\mathrm{a})$-schéma à $(1+1)$ photons conduisant à l'ionisation laser résonnante de $\mathrm{Si}^{\circ}$; (b)-spectre de photoionisation des atomes de $\mathrm{Si}^{\circ}$

\subsection{Distributions temps de vol}

Le traitement numérique des distributions de temps de vol expérimentaux (cf figure 2-(a) et (b)) par des distributions de Maxwell-Boltzmann [4] sans vitesse de centre de masse nous permet de déterminer l'énergie cinétique des atomes désorbés. Pour le domaine de fluence allant de 300 à $700 \mathrm{~mJ} / \mathrm{cm}^{2}$, ces énergies augmentent de 0,16 à $0,45 \mathrm{eV}$.

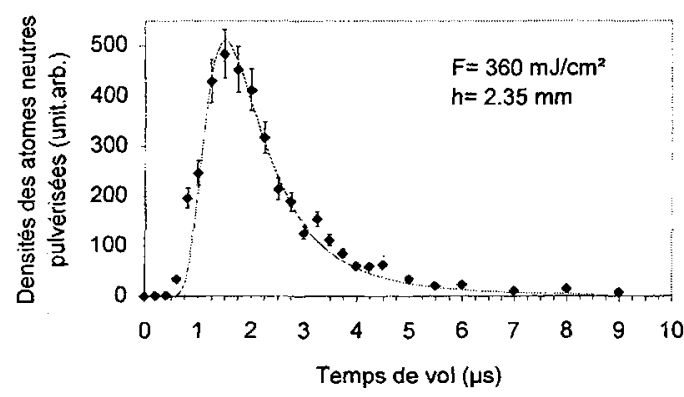

(a)

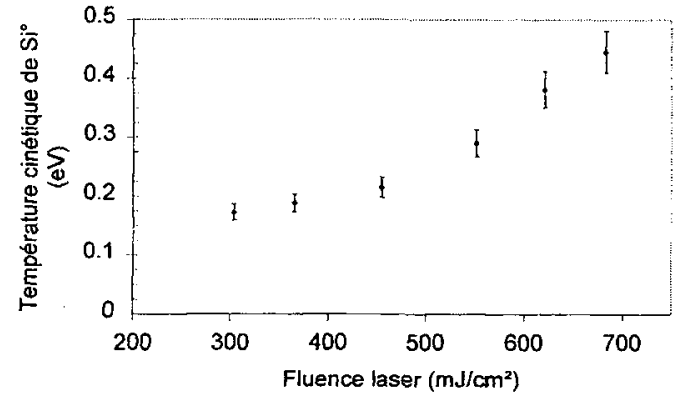

(b)

Figure 2. Temps de vol des atomes de silicium : (a)-ajustement d'une distribution expérimentale $\left(\mathrm{F}=360 \mathrm{~mJ} / \mathrm{cm}^{2} ; \mathrm{h}=2,35\right.$ $\mathrm{mm})$ par une distribution de Maxwell-Boltzmann $(\mathrm{kT}=0,19 \mathrm{eV})(\mathrm{b})$-évolution de la température cinétique des atomes neutres en fonction de la fluence laser.

D'après nos résultats expérimentaux, les températures cinétiques des atomes désorbés sont élevées (supérieures à la température de fusion du silicium). Il semble donc que la désorption du silicium ne soit pas d'origine purement thermique, et que des phénomènes induits par transitions électroniques soient possibles dans le processus de désorption.

\section{CONCLUSIONS}

Pour la première fois, nous avons utilisé la post-ionisation laser du silicium neutre pour obtenir des renseignements sur la dynamique de désorption laser d'une surface de silicium. Des expériences complémentaires à plus faibles fluences sont en cours de réalisation afin de mieux comprendre les mécanismes intervenant lors de l'interaction laser UV/ surface de silicium. La suite de l'étude va consister en l'analyse des produits de désorption issus de l'interaction $\mathrm{NH}_{3}-\mathrm{Si}$ / laser UV par mesure de temps de vol.

\section{Références}

1. Q.Zhuang, K.Ishigoh, K.Tanaka, K.Kawano et R.Nakata, Jpn. J. Appl. Vol. 34 (1995) pp.L248-L251.

2. L.Chen, V.Liberman, J.A.O'Neil, Z.Whu et R.M Osgood Jr., J. Vac. Sci. Technol. A, 6, 1426 (1988).

3. L.Patrone, thèse de l'Université de la Mediterranée- Aix-Marseille II -France- (2000)

4. R.Kelly et R.W Dreyfus, Surface Science 198 (1988), 263-276. 